\title{
EXISTENCE THEOREMS FOR OPTIMAL PROBLEMS WITH VECTOR-VALUED COST FUNCTION $\left(^{(}\right)$
}

\author{
BY \\ CZESLAW OLECH
}

Introduction. In this paper we consider the following optimal control problem. Consider a control system

$$
\dot{y}=f(t, y, u),
$$

where $f$ maps $J_{0} \times Y \times E$ into $Y, J_{0}=\left[a_{0}, b_{0}\right]$ is an interval, and $Y, E$ are Euclidean spaces. By a solution of $(0.1)$ is meant a triple $(J, y, u)$, where $J \subset J_{0}$ is an interval, $y: J \rightarrow Y$ is absolutely continuous, $u: J \rightarrow E$ is Lebesgue measurable, and $\dot{y}(t)$ $=f(t, y(t), u(t)), u(t) \in U(t, y(t))$ almost everywhere (a.e.) in $J$. The control domain $U$ is a map from $J_{0} \times Y$ into subsets of $E$.

Besides the system (0.1), a "cost function"

$$
I(J, y, u)=\int_{J} g(t, y(t), u(t)) d t
$$

is given, where $(J, y, u)$ is a solution of $(0.1)$ and $g$ maps $J_{0} \times Y \times E$ into another Euclidean space $X$. An order " $\leqq$ " is given in $X$ and with respect to which the positive cone $C$ is convex and closed and $C-C=\left\{x \mid x=c_{1}-c_{2}, c_{1}, c_{2} \in C\right\}=X$.

The problem is to minimize $I$ (with respect to the order in $X$ ) in a given class $\Omega$ of solutions of (0.1). The class $\Omega$ may be determined, for instance, by boundary conditions $y\left(a_{0}\right) \in A_{0}, y\left(b_{0}\right) \in B_{0}$ where $A_{0}, B_{0}$ are given fixed sets. Since the order is in general not total, we are thus looking for minimal points of $I(\Omega)$ rather than an absolute minimum; that is, we want to find $\omega^{*} \in \Omega$ such that for each $\omega \in \Omega$ the inequality $I(\omega) \leqq I\left(\omega^{*}\right)$ implies $I\left(\omega^{*}\right) \leqq I(\omega)$. Such an $\omega^{*}$ will be called an optimal solution.

This problem (of minimizing a vector-valued function rather than a functional) has been formulated and discussed and is of some interest in applications. For referencès we refer the reader to the paper [2]. In this paper the authors give necessary conditions for an optimal solution.

In this paper we seek conditions on $f, g, U$ and $\Omega$ which guarantee the existence of an optimal solution.

In a recent paper, Lamberto Cesari [1] gave several theorems of this nature for the case when $X$ is one-dimensional. In contrast to preceding papers (cf. for

Received by the editors November 27, 1967.

( ${ }^{1}$ This research was supported in part by the National Aeronautics and Space Administration under Grant No. NGR-40-002-015, and in part by the United States Army Research Office, Durham, under Contract No. DA-31-124-ARO-D-270. 
example, Filippov [3], Marcus and Lee [11], Roxin [15], Ważewski [17]), noncompact control domains $U$ are considered in [1], which allows the author to give a unified and very general existence theory applying to both the Pontrjagin optimal control problem and to the classical Lagrange problem in the calculus of variations. The present paper is strongly inspired by Cesari's recent work and it presents some generalizations of some of his theorems in [1]. The generalizations are twofold. On the one hand, we treat the problem with a vector-valued cost function whereas Cesari considered the scalar case. On the other hand, we are able to relax some of the regularity conditions on $f, g$ and $U$ assumed in [1]. To use an analogy from ordinary differential equations, the continuity requirements are replaced by Carathéodory's type assumptions. For a more detailed account of the relation between our results and those of Cesari see Remark 2 of $\S 3$.

However, the novelty of this paper lies perhaps more in its approach than in its results. In particular, the author believes that the lemma of $\S 1$ is by itself of some interest.

To prove the existence of a minimal point of $I(\Omega)$ one shows first that the closure $\operatorname{cl} I(\Omega)$ has such a point. This gives a minimizing sequence $\left\{\omega_{k}\right\} \subset \Omega$; and now we want to connect with such a sequence an element $\omega^{*}$ of $\Omega$ such that $I\left(\omega^{*}\right) \leqq$ $\lim _{k \rightarrow \infty} I\left(\omega_{k}\right)=$ a minimal point of $\mathrm{cl} I(\Omega)$. This is usually done by establishing a certain compactness property for $\Omega$ and continuity for $I$. For example, Filippov's existence theorem [3], [4] can be based upon the following fact (cf. [10]). Suppose $\Omega$ is the set of absolutely continuous functions $y: J \rightarrow Y$ uniformly bounded on $J$ (by a fixed constant) and such that $\dot{y}(t) \in P(t)$ a.e. in $J$, where $P(t)$ is a convex and closed subset of $Y$ and $\max _{p \in P(t)}\|p\|$ is bounded by an integrable function. Then $\Omega$ is compact in the uniform convergence topology. The lemma of $\S 1$ is a suitable extension of the above fact so that it applies also to the noncompact case considered by Cesari. To be more specific, the boundedness assumption on $P$, which can be equivalently expressed as the statement for each $c \in Y$ there is an integrable $\phi_{c}: J \rightarrow R$ such that

$$
\max _{p \in P(t)}\langle c, p\rangle \leqq \phi_{c}(t) \quad \text { a.e. in } J,
$$

is replaced in the lemma by the same condition but restricted to $c$ from an open convex cone. This allows $P(t)$ to be unbounded but only in certain directions. In this case $\Omega$ is no longer compact but still each sequence contains a convergent subsequence (pointwise, not uniformly) to a function which is not in general absolutely continuous but is of bounded variation.

The absolutely continuous part of the limit belongs to $\Omega$ while the singular part is positive and nondecreasing. The latter remark replacing the essence of the usual lower semicontinuity argument in the calculus of variations is contained in Cesari's paper (cf. [1, Closure 'Theorem II, p. 386, and the proof of Existence Theorem I, p. 391]). The lemma exploits this idea further, expressing it explicitly as well as in a more general setting. 
We believe our approach is different and more geometric. For instance, the socalled growth condition in the existence theorems of the calculus of variations appearing also in [1] is replaced here by a geometrical assumption expressed in terms of "the shape" of certain convex sets. Also, the proof of the lemma is based on a characterization of convex closed set which does not contain a line given in [7] and on some simple ideas used in [10].

The optimal problem described above is equivalent to an optimization problem for a system with multivalued right-hand side, or in Ważewski's terminology [17] an orientor field. This latter problem is treated in $\$ 2$.

In $\S 3$ we state and prove two existence theorems concerning the original optimal problem described above. These results are obtained as a combination of the results of $\$ 2$ and an appropriate extension of the so-called Filippov's implicit function lemma.

1. The principal lemma. Relevant to our considerations is the role played by closed convex sets which do not contain a line, and we begin by discussing some of their properties.

The following characterization of such sets is contained in [7], where a more general case of infinite-dimensional linear spaces is considered.

Proposition 1. Let $Z$ be a finite-dimensional Euclidean space and $P$ a proper subset of $Z$. Then the following two conditions are equivalent:

(i) $P$ is closed, convex and does not contain a line.

(ii) For each dense subset $D$ of $Z$ the equality holds

$$
P=\bigcap_{d \in D}\left\{z \mid\langle d, z\rangle \leqq \sup _{p \in P}\langle d, p\rangle\right\}
$$

where $\langle,>$ is the scalar product in $Z$.

For our purpose we will need a modification of (ii). For each subset $P$ of $Z$ define

$$
C_{P}=\{a \mid p+\lambda a \in P \text { for each } p \in P \text { and } \lambda \geqq 0\} .
$$

The set $C_{P}$ is not empty (always contains 0 ) and is a cone. Indeed, it is clear by definition (1.2) that if $a \in C_{P}$ and $\lambda \geqq 0$ then $\lambda a \in C_{P}$; thus $\lambda C_{P} \subset C_{P}$ for each $\lambda>0$. The set $C_{P}$ is called the asymptotic cone of $P$. If $P$ is closed then $C_{P}$ is closed, and if $P$ is convex then so is $C_{P}$. Finally, if $P$ is closed and convex, the cone $C_{P}$ is proper (does not contain a line: $\left.C_{P} \cap\left(-C_{P}\right)=\{0\}\right)$ if and only if $P$ does not contain a line.

Summing up, we can state that, for a closed convex set $P$ which does not contain a line, the asymptotic cone $C_{P}$ is closed convex and proper.

Consider now the polar $C_{P}^{0}$ of $C_{P}$; that is, the set

$$
C_{P}^{0}=\left\{c \mid\langle c, a\rangle \leqq 0 \text { for each } a \in C_{P}\right\} .
$$

Note that the supremum in the right-hand side of (1.1) can be finite only if $d \in C_{P}^{0}$. Thus (1.1) still holds if we replace $D$ by $D \cap C_{P}^{0}$ or any dense subset of 
$C_{P}^{0}$. Since in (ii) the relation (1.1) is supposed to hold for each dense $D$, it follows that if (ii) is true then $C_{P}^{0}$ has a nonempty interior and $D$ can be replaced in (1.1) by $D \cap$ int $C_{P}^{0}$.

On the other hand, if int $C_{P}^{0}$ is not empty and for each dense $D \subset Z$

$$
P=\bigcap_{d \in D \cap i n t C_{P}^{0}}\left\{z \mid\langle d, z\rangle \leqq \sup _{p \in P}\langle d, p\rangle\right\}
$$

then (1.1) holds. Indeed, for each $D$ the set $P$ is contained in the right-hand side of (1.1) and the latter is contained in the right-hand side of (1.4) and therefore is in $P$. Hence we have (1.1) and have shown the following:

Proposition 1'. If $P$ is a proper subset of $Z$, then (i) is equivalent to

(ii') $C_{P}^{0}$ has a nonempty interior and (1.4) holds for each dense subset $D$ of $Z$.

Note that if $P$ satisfies (i), then $\max _{p \in P}\langle d, p\rangle$ exists for each $d \in$ int $C^{0} \mid\{0\}$ and is finite. Indeed, the set

$$
P_{d, \alpha}=\{z \mid\langle d, z\rangle \geqq \alpha\} \cap P
$$

is compact for each $\alpha$ if $d \in$ int $C_{P}^{0}$. If the set $P_{d, \alpha}$ were unbounded, there would exist an $a \neq 0$ such that $p+\lambda a \in P_{d, \alpha}$ for each $p \in P_{d, \alpha}$ and $\lambda>0$. Thus $a \in C_{P_{d, \alpha}} \subset C_{P}$. But $\langle d, p+\lambda a\rangle \geqq \alpha$ for each $\lambda>0$ which implies that $\langle d, a\rangle \geqq 0$. The latter inequality contradicts the assumption that $d \in$ int $C^{0} \mid\{0\}$. Therefore $P_{d, \alpha}$ is bounded, and since it is always closed, it is compact, and the existence of $\max _{p \in P}\langle d, p\rangle$ follows. Hence in (1.4) 'sup' can be replaced by 'max'.

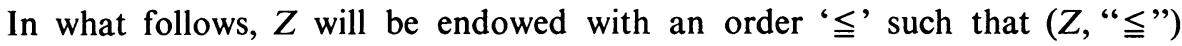
forms an ordered vector space and such that the positive cone $C$ is closed and convex. (Note that the same "ฏ" is used to denote the usual inequality between scalars.)

Let $X=C-C=\left\{z \mid z=c_{1}-c_{2}, c_{1}, c_{2} \in C\right\}$. The set $X$ is a closed subspace of $Z$. By $Y$ we denote the orthogonal complement of $X$. In particular, either $X$ or $Y$ can be zero-dimensional. In other words, we do not exclude $C=\{0\}$ or $C-C=Z$. Of course, $Z$ is the direct sum of $X$ and $Y$ and therefore each $z \in Z$ can be uniquely represented as the sum $x+y$ where $x \in X$ and $y \in Y$. If a letter other than $z$, say $c$, is used to denote a point in $Z$, then $c_{x}$ and $c_{y}$ will stand for the unique components of $c$ in $X$ and $Y$ respectively. Now we can state our basic lemma.

Lemma. Let $P$ be a map of an interval $J=[a, b]$ into closed convex subsets of $Z$. Assume that

$$
C_{P(t)}=C \text { for each } t \in J
$$

and that for each $c \in$ int $C^{0} \mid\{0\}$ there is an integrable $\phi_{c}: J \rightarrow R$ such that

$$
\max _{p \in P(t)}\langle c, p\rangle \leqq \phi_{c}(t),
$$

where $C^{0}$ is the polar of $C$. 
Let $z_{k}: J \rightarrow Z$ be absolutely continuous and uniformly bounded on $J, k=1,2, \ldots$ Assume that for each $k$

$$
\dot{z}_{k}(t) \in P(t) \text { a.e. in } J \text {. }
$$

Under these assumptions there is a subsequence $z_{k_{i}}, i=1,2, \ldots$, converging everywhere in $J$ to a function $z+v$ where:

$1^{\circ} z$ is absolutely continuous and

$$
\dot{z}(t) \in P(t) \text { a.e. in } J \text {. }
$$

$2^{\circ} v$ is singular and increasing; that is,

$$
\dot{v}(t)=0 \text { a.e. in } J \text { and } v(s) \leqq v(t) \text { if } s \leqq t .
$$

$3^{\circ}$ If $y_{k_{i}}(t)$ denotes the Y-component of $z_{k_{i}}(t)$, then

$$
y_{k_{i}}(t) \rightarrow y(t) \text { uniformly in } J,
$$

where $y(t)$ is the $Y$-component of $z(t)$.

The proof of the lemma will be preceded by a proposition, which essentially is the one-dimensional counterpart of the lemma.

Proposition 2. Let $\alpha_{k}: J \rightarrow R$ be absolutely continuous and uniformly bounded, $k=1,2, \ldots$ Assume that

$$
\dot{\alpha}_{k}(t) \leqq \lambda(t) \leqq \phi(t) \quad \text { a.e. in } J,
$$

where $\phi$ is integrable.

Then there exists a subsequence $\left\{\alpha_{k_{i}}\right\}$ converging everywhere to a function $\alpha+\beta$, where $\alpha: J \rightarrow R$ is absolutely continuous and

$$
\dot{\alpha}(t) \leqq \lambda(t) \text { a.e. in } J,
$$

and $\beta: J \rightarrow R$ is singular and nonincreasing; that is

$$
\beta(t)=0 \text { a.e. in } J \text { and } \beta(t) \geqq \beta(s) \text { if } t \leqq s .
$$

Moreover, for each $\varepsilon>0$ there is an $i_{0}$ such that

$$
\beta(b)-\varepsilon \leqq \alpha_{k_{i}}(t)-\alpha(t) \leqq \beta(a)+\varepsilon \quad \text { if } i \geqq i_{0} \text { and } t \in J .
$$

Proof. Put $\gamma(t)=\sup _{k} \dot{\alpha}_{k}(t)$ and $\delta_{k}(t)=\alpha_{k}(t)-\int_{a}^{t} \gamma(\tau) d \tau$. By (1.11), $\gamma(t) \leqq \lambda(t)$ a.e. in $J$ and $\gamma$ is integrable. Since $\left\{\alpha_{k}\right\}$ is uniformly bounded, so is $\left\{\delta_{k}\right\}$. By definition, the $\delta_{k}$ are nonincreasing for each $k ; \dot{\delta}_{k}(t) \leqq 0$ a.e. in $J$ for each $k$. Thus an everywhere convergent subsequence $\left\{\delta_{k_{i}}\right\}$ can be chosen and the limit function is also nonincreasing. As such, by the canonical decomposition theorem, it can be represented as the sum $\delta+\beta$, where $\delta$ is absolutely continuous, $\beta$ is singular, and both are nonincreasing. Thus the corresponding sequence $\left\{\alpha_{k_{1}}\right\}$ converges everywhere to $\alpha+\beta$ where $\alpha(t)=\delta(t)+\int_{a}^{t} \gamma(\tau) d \tau$. Hence $\alpha$ is absolutely continuous and $\dot{\alpha}(t)=\dot{\delta}(t)+\gamma(t) \leqq \gamma(t) \leqq \lambda(t)$. Thus (1.12) and $\beta$ satisfies (1.13). 
To prove the second part of Proposition 2, take an $\varepsilon>0$ and choose a partition $a=t_{0}<t_{1}<\cdots<t_{s}=b$ of $J$ such that $0 \leqq \delta\left(t_{j}\right)-\delta\left(t_{j+1}\right)<\varepsilon / 2$. Take $i_{0}$ so large that $\left|\delta_{k_{i}}\left(t_{j}\right)-\delta\left(t_{j}\right)-\beta\left(t_{j}\right)\right|<\varepsilon / 2$ for $i \geqq i_{0}$ and $j=0,1, \ldots, s$. These inequalities and monotonicity of $\delta_{k_{i}}, \delta$, and $\beta$ yield for $i \geqq i_{0}$ and $t_{j} \leqq t \leqq t_{j+1}$

$$
\begin{aligned}
\alpha_{k_{i}}(t)-\alpha(t) & =\delta_{k_{i}}(t)-\delta(t) \leqq \delta_{k_{i}}\left(t_{j}\right)-\delta\left(t_{j}\right)+\varepsilon / 2 \leqq \beta\left(t_{j}\right)+\varepsilon \\
& \leqq \beta(a)+\varepsilon
\end{aligned}
$$

and, similarly,

$$
\delta_{k_{j}}(t)-\delta(t) \geqq \delta_{k_{j}}\left(t_{j+1}\right)-\delta\left(t_{j+1}\right)-\varepsilon / 2 \geqq \beta\left(t_{j+1}\right)-\varepsilon \geqq \beta(b)-\varepsilon .
$$

Hence (1.14) follows, which completes the proof.

Proof of the Lemma. Let us take an arbitrary $d \in$ int $C^{0}$ and put $\alpha_{k}(t)=\left\langle d, z_{k}(t)\right\rangle$. Because of (1.6) and (1.7), $\left\{\alpha_{k}\right\}$ satisfies the assumptions of Proposition 2 with $\lambda(t)=\max _{p \in P(t)}\langle d, p\rangle$ and $\phi=\phi_{d}$. Therefore, there exists a subsequence $\left\{z_{k_{i}}\right\}$ such that

$$
\left\langle d, z_{k_{i}}(t)\right\rangle \rightarrow \alpha_{d}(t)+\beta_{d}(t), \quad t \in J,
$$

where $\alpha_{d}$ is absolutely continuous,

$$
\dot{\alpha}_{d}(t) \leqq \max _{p \in P(t)}\langle d, p\rangle \quad \text { a.e. in } J
$$

$\beta_{d}$ is singular and nonincreasing. Since int $C^{0}$ is not empty and open, there is a basis $\left\{d_{1}, \ldots, d_{n}\right\}$ of $Z$ contained in int $C^{0}$. Manifestly there is a subsequence $\left\{z_{k_{i}}\right\}$ such that (1.15) and (1.16) hold for each $d=d_{j}, j=1, \ldots, n$. But $\left\{d_{1}, \ldots, d_{n}\right\}$ is a basis and hence the subsequence $\left\{z_{k_{i}}\right\}$ itself has to be convergent. Therefore the limit function can be represented as a sum $z+v$ where $z(t)$ and $v(t)$ are unique solutions of the following systems, respectively

$$
\left\langle d_{j}, z(t)\right\rangle=\alpha_{d_{j}}(t), \quad\left\langle d_{j}, v(t)\right\rangle=\beta_{d_{j}}(t), \quad j=1, \ldots, n,
$$

and $z$ is absolutely continuous and $v$ is singular. Now since we have a convergent subsequence, (1.15) holds for each $d \in$ int $C^{0}$ and

$$
\alpha_{d}(t)=\langle d, z(t)\rangle, \quad \beta_{d}(t)=\langle d, v(t)\rangle .
$$

Clearly, $\alpha_{d}$ is absolutely continuous and $\beta_{d}$ is singular. Moreover, by Proposition 2 (1.16) holds and $\beta_{d}$ is nonincreasing for each $d \in$ int $C^{0}$. Hence

$$
\langle d, \dot{z}(t)\rangle \leqq \max _{p \in P(t)}\langle d, p\rangle \quad \text { a.e. in } J
$$

and

$$
\langle d, v(t)-v(s)\rangle \leqq 0 \quad \text { if } t<s .
$$

Both (1.17) and (1.18) hold for each $d \in$ int $C^{0}$. Now assumption (1.5) and (1.17) yield (1.8), while (1.18) implies that $v(t)-v(s) \in C$, that is, the second part of (1.9) holds.

To prove that the $Y$-component $y_{k_{i}}$ of $z_{k_{i}}$ converges uniformly to the $Y$ component $y$ of $z$, note that if $c \in$ int $C^{0} \cap X$ and $d \in Y$, then $d+\lambda c \in$ int $C^{0}$ for 
each $\lambda>0$. This follows from (1.2) and the fact that $\langle d+\lambda c, a\rangle=\lambda\langle c, a\rangle$ if $a \in C \subset X$ since $Y$ is the orthogonal complement of $X$. Let $\left\{d_{1}, \ldots, d_{s}\right\}$ be an orthonormal basis in $Y$, and let $c_{0}$ be a fixed point of int $C^{0} \cap X$. Without loss of generality we may assume that $v(a)=0$. Then by (1.9), $v(t) \in C \subset X$ for each $t \in J$. Take an $\varepsilon>0$ and choose $\lambda>0$ such that for each $i=1,2, \ldots$

$$
\lambda\left|\left\langle c_{0}, x_{k_{i}}(t)-x(t)\right\rangle\right|+\lambda\left|\left\langle c_{0}, v(b)\right\rangle\right|<\varepsilon, \quad t \in J .
$$

By (1.14) of Proposition 2, (1.19) and the equality

$$
\left\langle d_{j}+\lambda c_{0}, z_{k_{i}}(t)\right\rangle=\left\langle d_{j}, y_{k_{i}}(t)\right\rangle+\lambda\left\langle c_{0}, x_{k_{i}}(t)\right\rangle,
$$

we obtain the inequality

$$
\left|\left\langle d_{j}, y_{k_{i}}(t)-y(t)\right\rangle\right| \leqq 2 \varepsilon \quad \text { if } t \in J \text { and } i \geqq i_{0} .
$$

It is clear that $i_{0}$ can be chosen independently of $j$, since $j$ is from a finite set. Hence (1.20) implies uniform convergence of $y_{k_{i}}$ to $y$. Therefore the proof of the lemma is completed.

In the next section we will be dealing with solutions of generalized differential equations and they will not in general be defined on the same interval. Thus for our purposes we need to extend the lemma slightly.

Suppose a sequence $z_{k}: J_{k}=\left[a_{k}, b_{k}\right] \rightarrow Z, k=1,2, \ldots$, is given, where the domain interval may change with $k$. We denote this sequence by $\left\{z_{k}, J_{k}\right\}$. Assume that $J_{k} \subset J$ for each $k$.

Definition 1. We say that $\left\{z_{k}, J_{k}\right\}$ converges as $k \rightarrow \infty$ to $\left\{z_{0}, J_{0}\right\}, J_{0}=\left[a_{0}, b_{0}\right]$, pointwise (uniformly) if

$$
a_{k} \rightarrow a_{0}, \quad b_{k} \rightarrow b_{0} \quad \text { as } k \rightarrow \infty,
$$

and the sequence $\left\{\tilde{z}_{k}\right\}$ defined by

$$
\begin{aligned}
\tilde{z}_{k}(t) & =z_{k}\left(a_{k}\right), \quad a \leqq t \leqq a_{k}, \\
& =z_{k}(t), \quad a_{k} \leqq t \leqq b_{k}, \\
& =z_{k}\left(b_{k}\right), \quad b_{k} \leqq t \leqq b,
\end{aligned}
$$

converges pointwise (uniformly on $J$ ) to $\tilde{z}_{0}$, where $\tilde{z}_{0}$ is a similar extension of $\left(z_{0}, J_{0}\right)$.

REMARK 1. The lemma holds for the sequence $\left\{z_{k}, J_{k}\right\}$ when $J_{k} \subset J$ if (1.7) is replaced by $\dot{z}_{k}(t) \in P(t)$ a.e. in $J_{k}$ and convergence in the conclusion is in the sense of the above definition. In particular, for the convergent subsequence $\left\{z_{k_{i}}, J_{k_{i}}\right\}$ we have

$$
\begin{aligned}
& \left(a_{k_{i}}, y_{k_{i}}\left(a_{k_{i}}\right)\right) \rightarrow\left(a_{0}, y\left(a_{0}\right)\right), \\
& \left(b_{k_{i}}, y_{k_{i}}\left(b_{k_{i}}\right)\right) \rightarrow\left(b_{0}, y\left(b_{0}\right)\right),
\end{aligned}
$$

and

$$
x\left(b_{0}\right)-x\left(a_{0}\right) \leqq \lim _{i \rightarrow \infty}\left(x_{k_{i}}\left(b_{k_{i}}\right)-x_{k_{i}}\left(a_{k_{i}}\right)\right)
$$


To prove Remark 1, it is enough to notice that the modified set valued function

$$
\begin{aligned}
\tilde{P}_{\delta}(t) & =P(t) & & \text { if } t \in\left[a_{0}+\delta, b_{0}-\delta\right], \\
& =\operatorname{clco}[\{0\} \cup P(t)] & & \text { if } t \in J \backslash\left[a_{0}+\delta, b_{0}-\delta\right]
\end{aligned}
$$

(clco stands for convex closure) satisfies all assumptions of the lemma if $P(t)$ does and that the lemma can be applied to the sequence $\left\{\tilde{z}_{k}\right\}$ defined by (1.21). Since this can be done for each $\delta>0$ and since one chosen convergent subsequence is good for any other $\delta$, we may replace $\delta$ with zero, and thus (1.7) holds on $J_{0}$. Now (1.22) follows from the uniform convergence of $\tilde{y}_{k_{i}}$, while (1.23) is a consequence of the monotonicity of $\bar{v}$.

REMARK 2. If in the above we assume that $x_{k}\left(a_{k}\right)=0$, then $\tilde{x}_{k}(t)=0$ if $t \in\left[a_{0}, a_{k}\right]$ for each $k$ and thus also the $x$-part of the limit function $\tilde{x}(t)+\tilde{v}(t)=0$ if $a \leqq t<a_{0}$. Hence, fixing $\tilde{v}(a)=0$, we conclude by continuity of $\tilde{x}$ that $\tilde{x}\left(a_{0}\right)=0$. Hence, the limit function satisfies the same initial condition.

Remark 3. If $C=\{0\}$ then $Z=Y$ and we have the case discussed in the introduction: any bounded sequence contains a uniformly convergent subsequence. This special case of the lemma is given in [10] (cf. also [4] and [14]). Perhaps it is worthwhile to point out that the set valued function $P$ can be eliminated from both the assumptions and the conclusion of the lemma. In other words, if we assume that a sequence $\left\{z_{k}\right\}$ of absolutely continuous functions is bounded and, for each $c \in$ int $C^{0},\left\langle c, \dot{z}_{k}(t)\right\rangle$ is bounded by an integrable function independent of $k$, then the conclusion of the lemma remains valid with (1.8) deleted.

REMARK 4. If the sequence $\left\{z_{k}\right\}$ in the lemma converges pointwise to an absolutely continuous function, then the convergence is uniform. Indeed, if in Proposition 2 the singular part $\beta$ is zero, then by (1.14) $\alpha_{k_{i}} \rightarrow \alpha(t)$ uniformly. Thus, under our assumption $\beta_{d}(t) \equiv 0$ in (1.15) and the convergence is uniform for each $d \in$ int $C^{0}$. Hence, the singular part $v$ has to be equal to zero and $z_{k}(t) \rightarrow z(t)$ uniformly. In fact, the same statement could be proved if the limit function is continuous (the singular part is continuous). For that purpose part (1.14) of Proposition 2 should be changed.

The above discussion brings to mind the classical Dini theorem. In fact Proposition 2 is a combination of Helly's theorem and (1.14) is "almost" the Dini theorem. Therefore our lemma could be considered as generalization of those two results.

Finally, let us mention that the integrability of functions $\phi_{c}$ is not essential for the validity of this remark. Indeed as follows from Theorem I of [10] and Proposition 1 ', it is enough to assume that $\phi_{d}$ is locally integrable; that is, for almost all $t \in J$ there is a neighiorhood of $t$ on which $\phi_{d}$ is integrable. However the lemma itself is no longer true if $\phi_{d}$ is not integrable.

2. Existence theorems for orientor fields. Consider a map $Q: J_{0} \times Z \rightarrow 2^{Z}$ $\left(2^{Z}\right.$ stands for the set of all subsets of $Z$ ). The following expression

$$
\dot{z} \in Q(t, z)
$$


is called an orientor field or a differential equation with multi-valued right-hand side. By a solution of (2.1) we will mean a pair $(J, z)$ where $J=[a, b] \subset J_{0}$ is an interval, $z$ is an absolutely continuous function from $J$ into $Z$ and (2.1) is satisfied a.e. in $J$; that is, $\dot{z}(t) \in Q(t, z(t))$ a.e. in $J$.

The optimal problem we described in the introduction can be reduced to the following optimization problem for (2.1). As before let $C$ be a closed, convex and proper cone in $Z, X=C-C$ and let $Y$ be the orthogonal complement of $X$. For any solution $\omega=(J, z)$ of $(2.1)$ define

$$
I(J, z)=x(b)-x(a),
$$

where $x(t)$ denotes the $X$ component of $z(t)$.

The problem in question is to minimize $I$ in a given class $\Omega$ of solutions of (2.1). More precisely, we want conditions which will imply for a given $\Omega$ the existence of an optimal solution; that is, an $\omega^{*} \in \Omega$ such that for each $\omega \in \Omega$ the inequality $I(\omega) \leqq I\left(\omega^{*}\right)$ implies $I\left(\omega^{*}\right)=I(\omega)$, where the order is that induced by the cone $C$.

Naturally, $\Omega$ cannot be arbitrary and we impose upon $\Omega$ the following conditions. Since the conditions which follow are different for the $Y$ and $X$ parts of the solution, we shall in future denote a solution by $(J, x, y)$ and mean that $x: J \rightarrow X$, $y: J \rightarrow Y$ are both absolutely continuous and that $z(t)=x(t)+y(t)$ satisfies $(2.1)$ on $J$. Recall that $J=[a, b]$.

(I) If $(J, x, y) \in \Omega$, then $x(a)=0$.

(II) If $(J, x, y) \in \Omega,(J, \bar{x}, y)$ is a solution of $(2.1)$ and $\bar{x}(a)=0$, then $(J, \bar{x}, y) \in \Omega$.

(III) If $\left(J_{k}, x_{k}, y_{k}\right) \in \Omega, k=1,2, \ldots,\left(J_{0}, x_{0}, y_{0}\right)$ is a solution of $(2.1),\left(J_{k}, y_{k}\right)$ $\rightarrow\left(J_{0}, y_{0}\right)$ uniformly (cf. Definition 1) and if $x_{0}(a)=0$, then $\left(J_{0}, x_{0}, y_{0}\right) \in \Omega$.

(IVa) There is a constant $M>0$ such that $\|y(t)\| \leqq M$ for each $(J, x, y) \in \Omega$ and for each $t \in J$.

(IVb) There is a constant $M>0$ with the property that for each $(J, x, y) \in \Omega$ there is $t \in J$ such that $\|y(t)\| \leqq M$.

The above restrictions on $\Omega$ are motivated by applications. In fact the $y$ part of $z$ will be a solution of system (0.1) while $x(b)$ is the value of (0.2). Condition (III) is replaced in more concrete cases by a boundary value type condition; for example, the end points $(a, y(a))$ and $(b, y(b))$ are tied to compact sets. In this case (IVb) will automatically be satisfied. We face condition (IVa), for example, when we are restricted to solutions of $(0.1)$ whose graphs are in a compact set. For simplicity we shall call a class $\Omega$ admissible if (I), (II), (III) and (IVb) hold and bounded admissible if (IVb) is replaced by (IVa).

The two theorems which follow give sufficient conditions for the existence of an optimal element in a bounded admissible $\Omega$ and an admissible $\Omega$, respectively.

Below, by an upper semicontinuous (u.s.c.) map $Q: Y \rightarrow 2^{Z}$ ( $Y, Z$-topological spaces in general), we mean simply that the graph of $Q$ in $Y \times Z$ is closed (cf. Kuratowski [8]). In particular, the map $Q$ in (2.1) is u.s.c. in $z$ for each fixed $t$ if 
for any $z_{k} \rightarrow z_{0}$ and $q_{k} \rightarrow q_{0}$ such that $q_{k} \in Q\left(t, z_{k}\right)$ we can conclude that $q_{0} \in$ $Q\left(t, z_{0}\right)$. If $Q$ is u.s.c., then values of $Q$ are closed sets.

THEOREM 1. Assume that $Q$ in (2.1) is u.s.c. in z for each fixed t, values of $Q$ are convex sets, and the asymptotic cone of $Q(t, z)$ is constant and equal to $C$; that is,

$$
C_{Q(t, z)}=C=\text { const. }
$$

In addition, assume that

$$
Q(t, \tilde{z}) \subset Q(t, \bar{z}) \quad \text { if } \bar{z} \leqq \tilde{z}
$$

and that for each $d \in$ int $C^{0}$ and positive $r$ there exists an integrable $\phi_{d}(t, r)$ such that

$$
\max _{q \in Q(t, z)}\langle d, q\rangle \leqq \phi_{d}(t, r) \quad \text { if }\|y\| \leqq r
$$

where $y$ is the $Y$-component of $z$.

Under these assumptions any bounded admissible class $\Omega$ contains an optimal element.

If condition (IVa) for $\Omega$ is replaced by the weaker condition (IVb), then the conclusion is still true provided some additional restrictions on $\phi_{d}$ in (2.5) are imposed. Namely, we have the following:

THEOREM 2. Let $Q$ in (2.1) satisfy all the assumptions of Theorem 1. In addition, assume there is $c \in\left(\right.$ int $\left.C^{0}\right) \cap X$ such that one of the following conditions holds:

(A) The function $\phi_{c}(t, r) \cdot$ in (2.5) does not depend on $r$ and there is an $\eta>0$ such that for each $d \in Y,\|d\|=1$, the function $\phi_{d+n c}$ is linear in $r$.

(B) The function $\phi_{c}(t, r)$ as well as $\phi_{n c+d}$ is linear in $r$ for each $d \in Y,\|d\|=1$, and $\eta \in\left(0, \eta_{0}\right], \eta_{0}>0$, and if $\phi_{n c+d}(t, r)=\phi_{n c+d}(t)+r \psi_{n c+d}(t)$, then

$$
\int_{J_{0}} \psi_{\eta c+d}(t) \leqq m<+\infty, \quad \text { if }\|d\|=1, \eta \in\left(0, \eta_{0}\right]
$$

Then any admissible class $\Omega$ of solutions of (2.1) contains an optimal element.

REMARK 1. In applications of these theorems to the problem discussed in the introduction, condition (2.4) is automatically satisfied since the set valued function is independent of " $x$ " and depends only on $\|y\|$. Thus $Q(t, \bar{z})=Q(t, \tilde{z})$ for any $\bar{z} \leqq \tilde{z}$.

Before proving Theorems 1 and 2 we show the following:

Proposition 3. Assume that the map $Q: Z \rightarrow 2^{Z}$ is u.s.c., the values of $Q$ are convex subsets of $Z$, the asymptotic cone $C_{Q(z)}=C=$ const. and that for each $r>0$ and $d \in$ int $C^{0}$

$$
\sup _{\|z\|} \sup _{q \in Q(z)}\langle d, q\rangle \text { is finite. }
$$


Then the map $Q$ has the following property (property $(Q)$ of Cesari [1]):

$$
Q\left(z_{0}\right)=\bigcap_{r>0} \operatorname{clco} \bigcup_{\left\|z-z_{0}\right\| \leqq r} Q(z) .
$$

Proof. Let us select $r_{0}$ and $z_{0}$ so that $\left\|z_{0}\right\|<r_{0}$. It is clear that the left-hand side of (2.8) is contained in the right-hand side. To prove the opposite take $q_{0} \notin Q\left(z_{0}\right)$. Since $C$ is assumed to be the asymptotic cone of $Q\left(z_{0}\right)$ it follows from Proposition 1' that there is a $d_{0} \in$ int $C^{0}$ and an $\varepsilon>0$ such that

$$
\max _{q \in Q(z 0)}\left\langle d_{0}, q\right\rangle\left\langle\left\langle d_{0}, q_{0}\right\rangle-\varepsilon .\right.
$$

On the other hand, for the same reasons

$$
Q(z) \cap\left\{q \mid\left\langle d_{0}, q\right\rangle \geqq\left\langle d_{0}, q_{0}\right\rangle-\varepsilon\right\}
$$

is compact for each $z$ whose norm is bounded by $r_{0}$. We want to show that (2.10) is empty if $\left\|z-z_{0}\right\|<r_{1}$ and $r_{1}$ is small enough. Suppose the contrary. Then there would exist sequences $z_{n} \rightarrow z_{0}$ and $q_{n} \in Q\left(z_{n}\right)$ such that

$$
\left\langle d_{0}, q_{n}\right\rangle \rightarrow \gamma \geqq\left\langle d_{0}, q_{0}\right\rangle-\varepsilon .
$$

If $q_{n}$ were convergent or contained a convergent subsequence, then we would have a contradiction with upper semicontinuity of $Q$ because of (2.9). Therefore $\left\|q_{n}\right\|$ $\rightarrow \infty$. But in that case, since $C^{0}$ has no empty interior, there exists $d_{*} \in$ int $C^{0}$ such that $\lim \sup \left\langle d_{*}, q_{n}\right\rangle$ is infinite which contradicts (2.7). Hence there is an $r_{1}>0$ such that for $\left\|z-z_{0}\right\|<r_{1}$ the set (2.10) is empty which shows that $q_{0}$ does not belong to the right-hand side of (2.8) and completes the proof.

Remark 3. Note that under the assumptions of Proposition 2 the function $\sup _{q \in Q(z)}\langle d, q\rangle$ is u.s.c. in $z$ for each $d$ in int $C^{0}$. This is an immediate consequence of (2.8). Note also that it suffices to assume (2.7) for $d$ from any fixed dense subset $D$ of int $C^{0}$. The last remark and Proposition 3 give the following.

COROLlary. If $Q: J \times Z \rightarrow Z$ satisfies the assumptions of Theorem 1 , then there is a subset $N \subset J$ of measure zero such that $Q(t, z)$ has property (2.8) in $z$ if $t \in J \backslash N$.

Indeed, fixing a denumerable dense subset $D$ of int $C^{0}$ and a sequence $r_{n} \rightarrow \infty$, there is a set $N$ of measure zero such that $\phi_{d}(t, r)$ is finite if $t \in J \backslash N, r \in\left\{r_{n}\right\}$, and $d \in D$. Hence (2.7) holds for any fixed $t$ from $J \backslash N$.

Proof of Theorem 1. Let $\Omega_{1} \subset \Omega$ be such that $I\left(\Omega_{1}\right)$ is totally ordered by "ฏ". By Kuratowski-Zorn lemma, Theorem 1 will be proved if we show that for each such $\Omega_{1}$ there is $\bar{\omega} \in \Omega$ such that $I(\bar{\omega}) \leqq I(\omega)$ for each $\omega \in \Omega_{1}$.

Let $p \in I\left(\Omega_{1}\right)$ be arbitrary. Since $I\left(\Omega_{1}\right)$ is totally ordered,

$$
I\left(\Omega_{1}\right) \subset p+(C \cup(-C)) .
$$

In particular, if we take an arbitrary $d \in$ int $C^{0} \mid\{0\}$ and denote by $\pi(d, p)$ the hyperplane passing through $p$ perpendicular to $d$, then because of (2.11)

$$
I\left(\Omega_{1}\right) \cap \pi(d, p)=\{p\} .
$$


The latter holds for each $p \in I\left(\Omega_{1}\right)$. Thus we can conclude that $I\left(\Omega_{1}\right)$ is a graph of a map from a subset of the line $\{z \mid z=\lambda d, \lambda \in R\}$ into the subspace $\{x \mid\langle x, d\rangle=0\}$ of $X$. Moreover, because of (2.11) and the closedness of $C$, the map in question satisfies a Lipschitz condition and therefore is continuous. Thus also the closure cl $I\left(\Omega_{1}\right)$ is a totally ordered set, since it is again a graph of a Lipschitzian map. On the other hand, by $(2.5)$ and the integrability of $\phi_{d}(\cdot, M)$ we have

$$
\sup _{p \in I\left(\Omega_{1}\right)}\langle d, p\rangle \leqq \int_{J_{0}} \phi_{d}(\tau, M) d \tau<+\infty
$$

where $M$ is the constant in (IVa). Therefore we can conclude that there is $p_{0} \in$ $\operatorname{cl}\left(I\left(\Omega_{1}\right)\right)$ such that $p_{0} \leqq p$ for each $p \in I\left(\Omega_{1}\right)$.

Now to complete the proof we need to show that there is $p_{*} \in I(\Omega)$ such that $p_{*} \leqq p$. Here is where the lemma is needed. Let $\left(J_{k}, x_{k}, y_{k}\right) \in \Omega_{1}$ and be such that

$$
I\left(J_{k}, x_{k}, y_{k}\right)=x_{k}\left(b_{k}\right) \rightarrow p_{0} \quad \text { as } k \rightarrow \infty .
$$

Put $z_{k}=x_{k}+y_{k}$ and

$$
P(t)=\operatorname{clco} \bigcup_{k \in K(t)} Q\left(t, z_{k}(t)\right)
$$

where $K(t)=\left\{k \mid t \in J_{k}\right\}$. In this way (2.15) defines a set valued map on $\bigcup J_{k}$ whose values are convex closed sets. It is a simple matter to check that the sequence $\left\{z_{k}\right\}$ and the map $P$ satisfies all assumptions of the lemma. Therefore the latter, together with Remark 1 and 2 of $\S 1$, imply that there exists a subsequence (for simplicity still denoted by $\left.\left(J_{k}, x_{k}, y_{k}\right)\right)$ converging pointwise to $\left(J_{*}, x_{*}+v, y_{*}\right)$ and such that $v(t) \geqq 0$,

$$
\begin{gathered}
\dot{z}_{*}(t)=\dot{x}_{*}(t)+\dot{y}_{*}(t) \in P(t) \text { a.e. in } J_{*}=\left[a_{*}, b_{*}\right], \\
\left(J_{k}, y_{k}\right) \rightarrow\left(J_{*}, y_{*}\right) \text { uniformly, } \\
x_{*}\left(a_{*}\right)=0,
\end{gathered}
$$

and

$$
x_{*}\left(b_{*}\right)=I\left(J_{*}, z_{*}\right) \leqq p_{0}=\lim _{k \rightarrow \infty} x_{k}\left(b_{k}\right) .
$$

To finish the proof it is sufficient now to prove that $\left(J_{*}, z_{*}\right)$ is a solution of (2.1) belonging to $\Omega$. The latter is a consequence of (2.17), (2.18) and condition (III) provided $\left(J_{*}, z_{*}\right)$ is a solution of $(2.1)$. This we will prove now. For that purpose define

$$
P_{j}(t)=\text { clco } \bigcup_{k \in K(t) ; k \geqq j} Q\left(t, z_{k}(t)\right) .
$$

For exactly the same reason as above, we have

$$
\dot{z}_{*}(t) \in P_{j}(t) \text { a.e. in } J_{*}, \quad j=1,2, \ldots
$$


The corollary to Proposition 3, $\left(2.16_{\mathrm{f}}\right)$, assumption (2.3), the pointwise convergence of $\left(J_{k}, z_{k}\right)$ to $\left(J_{*}, z_{*}+v\right)$, and the monotonicity of $v$ yield

$$
\dot{z}_{*}(t) \in \bigcap_{j=1} P_{j}(t)=Q\left(t, z_{*}(t)+v(t)\right) \subset Q\left(t, z_{*}(t)\right)
$$

for almost all $t \in J_{*}$. Therefore $\left(J_{*}, z_{*}\right)$ is a solution of (2.1) and the proof of Theorem 1 is completed.

Proof of Theorem 2. Let us take an arbitrary $\bar{\omega}=(\bar{J}, \bar{x}, \bar{y}) \in \Omega$ and put $\Omega_{0}$ $=\{\omega \in \Omega \mid I(\omega) \leqq I(\bar{\omega})\}$. It is clear that any minimal point of $I\left(\Omega_{0}\right)$ is also a minimal point of $I(\Omega)$. Thus it is enough to prove the existence of an optimal element in $\Omega_{0}$. We will do this by proving that $\Omega_{0}$ satisfies condition (IVa) and thus reduce the proof to Theorem 1 .

For that purpose let $\omega=(J, x, y) \in \Omega_{0}$ be arbitrary but fixed $(J=[a, b])$. Denote by

$$
\beta(t)=\|y(t)\| \quad \text { and } \quad \alpha(t)=\langle c, x(t)\rangle,
$$

where $c$ is the fixed point of (int $\left.C^{0} \mid\{0\}\right) \cap X$ in conditions (A) and (B). Notice that for each $d \in Y$ and any $\eta>0, \eta c+d \in$ int $C^{0}$. Suppose for some $\eta>0$ and each $d \in Y,\|d\|=1$, the function in the right-hand side of (2.5) is of the form $\phi_{d+n c}(t)$ $+r \psi_{d+n c}(t)$. Using the orthogonality of $X$ and $Y$ we get

$$
\langle d, \dot{y}(t)\rangle+\eta \dot{\alpha}(t) \leqq \phi_{d+\eta c}(t)+\beta(t) \psi_{d+n c}(t) .
$$

Since in both conditions (A) and (B) $\phi_{c}(t, r)$ is assumed to be linear, we may replace it by $\phi_{c}(t)+r \psi_{c}(t)$ and the analog of (2.22) for $d=0, \eta=1$ holds:

$$
\dot{\alpha}(t) \leqq \phi_{c}(t)+\beta(t) \psi_{c}(t) .
$$

Since (2.22) holds for both $d$ and $-d$, it holds also with $\langle d, \dot{y}(t)\rangle$ replaced by its absolute value, with obvious changes on the right-hand side. Therefore if $d_{1}, \ldots, d_{n}$ is an orthonormal basis in $Y$, we can deduce from (2.22) the following inequality

$$
\|\dot{y}(t)\|+\eta \dot{\alpha}(t) \leqq \lambda_{\eta}(t)+\mu_{\eta}(t) \beta(t), \quad \text { a.e. in } J,
$$

where

$$
\begin{aligned}
\lambda_{\eta}(t) & =\max _{l \leqq i \leqq n}\left\{\phi_{d_{i}+n c}(t), \phi_{-d_{i}+n c}(t)\right\}, \\
\mu_{\eta}(t) & =\max \left\{\psi_{d_{i}+n c}(t), \psi_{-d_{i}+n c}(t)\right\},
\end{aligned}
$$

and thus both are integrable on $J$. Since $(J, x, y) \in \Omega_{0} \subset \Omega$ we have by (I) and (2.21) that $\alpha(0)=0$, and from definition of $\Omega_{0}$ we get

$$
\alpha(b)=\langle c, x(b)\rangle \geqq\langle c, \bar{x}(b)\rangle=N_{0} .
$$

Moreover, (IVb) implies that there is a $t_{0} \in J$ such that

$$
0 \leqq \beta\left(t_{0}\right) \leqq M
$$


Taking into account the inequality $|\dot{\beta}(t)| \leqq\|\dot{y}(t)\|$ and (2.26) we get from (2.24) if $t \geqq t_{0}$ that

$$
\beta(t) \leqq \exp \left(\int_{t_{0}}^{t} \mu_{\eta}(\tau) d \tau\right)\left[M+\int_{t_{0}}^{t}\left(-\eta \dot{\alpha}(\tau)+\lambda_{\eta}(\tau)\right) \exp \left(-\int_{t_{0}}^{t} \mu_{\eta}(s) d s\right) d \tau\right],
$$

and if $t \leqq t_{0}$ that

$$
\beta(t) \leqq \exp \left(-\int_{t_{0}}^{t} \mu_{\eta}(\tau) d \tau\right)\left[M+\int_{t_{0}}^{t}\left(\eta \dot{\alpha}(\tau)-\lambda_{\eta}(\tau)\right) \exp \left(\int_{t_{0}}^{\tau} \mu_{\eta}(s) d s\right) d \tau\right] .
$$

Since $\lambda_{\eta}$ and $\mu_{\eta}$ are nonnegative we can, using $\left(2.27^{\prime}\right)$ and $\left(2.27^{\prime \prime}\right)$, estimate $\beta(t)$ as follows:

$$
\beta(t) \leqq \exp \left(\int_{J} \mu_{\eta}(\tau) d \tau\right)\left(M+\eta N_{1}+N_{2}\right), \quad t \in J_{0},
$$

where $N_{1}=-\int_{J_{0}} \delta_{-}(\tau) d \tau, \delta_{-}(t)=\min (0, \dot{\alpha}(t))$ and $N_{2}=\int_{J} \lambda_{n}(\tau) d \tau$. On the other hand, putting $\delta_{+}(t)=\max (0, \dot{\alpha}(t))$ we obtain by $(2.25)$

$$
\alpha(b)=\int_{J} \delta_{+}(\tau) d \tau+\int_{J} \delta_{-}(\tau) d \tau \geqq N_{0},
$$

and because of $(2.23)$

$$
\int_{J} \delta_{+}(\tau) d \tau \leqq \int_{J} \phi_{c}(\tau) d \tau+\int_{\tau} \beta(\tau) \psi_{c}(\tau) d \tau
$$

If (A) holds, then in (2.30) $\psi_{c} \equiv 0$ and thus $N_{1}$ is bounded by a constant depending only on $N_{0}$ and $\phi_{c}$. But (2.22) holds for some $\eta>0$, and therefore (2.28) is valid and it implies boundedness of $\beta(t)$ by a constant which does not depend on $\omega \in \Omega_{0}$. Hence $\Omega_{0}$ satisfies condition (IVa).

If (B) holds, then (2.28) can be used for $\eta \in\left(0, \eta_{0}\right], \eta_{0}>0$, and it is easy to see that (2.6) implies the analogous condition for $\mu_{\eta}$. Hence we have

$$
\int_{J_{0}} \mu_{\eta}(\tau) d \tau \leqq m_{0}<+\infty \text { if } \eta \in\left(0, \eta_{0}\right]
$$

and this plus (2.28)-(2.30) yield

$$
N_{1} \leqq \int_{J} \delta_{+}(\tau) d \tau-N_{0} \leqq \int_{J} \phi_{c}(\tau) d \tau+e^{m_{0}}\left(M+\eta N_{1}+N_{2}\right) \int_{J} \psi_{c}(\tau) d \tau-N_{0} .
$$

This in turn shows that if $\eta>0$ is small enough, then $N_{1}$ can be estimated by a constant depending only on $\eta$ and $c$ but not on a particular element of $\Omega_{0}$. Thus again $\Omega_{0}$ satisfies condition (IVa). Therefore in both cases we can apply Theorem 1 to $\Omega_{0}$ and this completes the proof of Theorem 2 .

EXAMPLE 1. In order to illustrate Theorem 1 and Theorem 2 and the difference between them, let us consider a very simple example in which both $X$ and $Y$ are one-dimensional. Then $Z$ is a plane, $C=\{(x, y) \in Z \mid x \geqq 0, y=0\}, C^{0}=\{(x, y) \mid x \leqq 0$, 
$y$ arbitrary\}, and suppose $J_{0}=[0,1]$. As $Q(t, z)$ we take the set valued function depending only on $y$ and given by

$$
Q(t, y)=\left\{q=\left(q_{x}, q_{y}\right) \mid q_{x} \geqq \alpha(t, y) q_{y}^{2}+\beta(t, y) q_{y}+\gamma(t, y)\right\},
$$

so that condition (2.4) is automatically satisfied (cf. Remark 1). The set (2.31) is convex provided $\alpha(t, y) \geqq 0$ and u.s.c. in $y$ if $\alpha, \beta, \gamma$ are continuous in $y$. The asymptotic cone $C_{Q(t, y)}$ is equal to $C$ if and only if $\alpha(t, y)>0$. It is clear that it is enough to assume $\alpha(t, y)>0$ for each $(t, y) \in(J \backslash N) \times Y$ where $N$ is a set of measure zero. Now the maximum in (2.5) can easily be calculated and is equal (for $(-\eta, \pm d$ ) $\in$ int $C^{0}$ ) to

$$
(\eta \beta(t, y) \mp d)^{2} / 4 \eta \alpha(t, y)-\eta \gamma(t, y) .
$$

Now assumption (2.5) says in this case that (2.32) is bounded on each compact subset of $Y$ by an integrable function of $t$ depending on $\eta$ and $d$.

Theorem 1 deals with the case when $\Omega$ is bounded (condition (IVa)) and that is why we are interested in having a bound for (2.32) on compact subsets of $Y$ only. One may say that in the case of Theorem 1 we assume (almost explicitly) two facts: $1^{\circ}$ the inf $x(b)$ for $(J, x, y) \in \Omega$ is finite, and $2^{\circ}$ it can be approached by a sequence with uniformly bounded $y$-components.

In the example we consider the additional assumption (A) of Theorem 2 is expressed by two inequalities:

$$
\beta^{2}(t, y) / 4 \alpha(t, y)-\gamma(t, y) \leqq \mu_{0}(t) \quad \text { if } y \in Y
$$

and for some $\eta>0$;

$$
(\eta \beta(t, y) \pm 1)^{2} / 4 \eta \alpha(t, y)-\eta \gamma(t, y) \leqq \mu_{\eta}(t)+|y| \lambda_{\eta}(t),
$$

where $\mu_{0}, \mu_{\eta}$, and $\lambda_{\eta}$ are integrable. So in this case we still assume $1^{\circ}$ (inequality (2.33)) but not $2^{\circ}$ since $\Omega$ is assumed to satisfy only (IVb). This is also the reason why (2.33) is assumed to hold for all $y$. Finally, in the case (B) condition (2.33) is relaxed by allowing on the right-hand side a term $\gamma_{0}(t)|y|$; so it is not obvious any more that $1^{\circ}$ holds and of course also $2^{\circ}$ has to be proved. Naturally this requires stronger assumption; this assumption is inequality (2.34), which now is supposed to hold for $\eta \in\left(0, \eta_{0}\right], \eta_{0}>0$; and besides, there is a constant $m_{0}<+\infty$ such that

$$
\int_{J_{0}} \lambda_{\eta}(\tau) d \tau \leqq m_{0}<+\infty \quad \text { if } \eta \in\left(0, \eta_{0}\right]
$$

It is easy to give many specific conditions on $\alpha, \beta$ and $\gamma$ such that one of the cases discussed takes place. We restrict ourself to two examples. In both we put $\beta \equiv 0$. First let

$$
\alpha(t, y)=t^{1-\varepsilon} /(1+|y|)
$$

so that the first term in (2.34) is $t^{-1+\varepsilon}(4 \eta)^{-1}(1+|y|)$; and therefore if $\gamma$ is supposed to be linear in $y$, then the inequality (2.34) holds for $\eta>0$. However, because of the 
term $(4 \eta)^{-1},(2.35)$ does not hold; therefore we are, in the case (A), assuming that (compare (2.33))

$$
+\gamma(t, y) \geqq \mu_{0}(t)
$$

$\mu_{0}$ integrable.

On the other hand, if we put

$$
\alpha(t, y)=t^{1-\varepsilon}
$$

then condition (B) holds if we assume

$$
\gamma(t, y) \geqq \mu_{0}(t)+\lambda_{0}(t)|y|,
$$

where $\mu_{0}, \lambda_{0}$ are integrable and possibly negative.

REMARK 2. Note that if $Q$ in (2.1) does not depend on $z$ then there is no difference between the assumptions of Theorem 1 and 2 , and they coincide with the assumptions concerning $P$ in the lemma. Therefore it follows from Theorem 2 that the following problem minimizes $x(b)$ in the class of absolutely continuous functions $z=(x, y): J \rightarrow Z$ such that (a) $x(a)=0$, (b) $y(a)=y_{1}, y(b)=y_{2}$, (c) $\dot{z}(t)$ $\in P(t)$, admits an optimal solution provided $P(t)$ satisfies the assumptions of the lemma.

In fact the above statement follows immediately from the lemma. The only thing one has to prove is that a minimizing sequence is bounded.

ExAmple 2. The aim of this example is to show that the statement in the above remark is no longer true if $\phi_{c}$ in the lemma is not integrable for some $c$. So let $Z$ be the plane $R^{2}, P(t)$ a closed and convex subset of $R^{2}$ such that for each $t \in[0,1]$

$$
\begin{gathered}
P(t) \cap\left\{(x, y) \in R^{2} \mid x \leqq 0\right\}=\{(0,0)\}, \\
C_{P(t)}=C=\{(x, y) \mid y=0, x \geqq 0\},
\end{gathered}
$$

and

$$
\max _{(x, y) \in P(t)}\left(-x+\eta_{n} y\right)=\phi_{n}(t)
$$

where $\eta_{n}>0, \eta_{n} \rightarrow 0, \phi_{n}(t)$ is positive, measurable but $\int_{0}^{1} \phi_{n}(t) d t=+\infty$. Finally, assume that for each $n=1,2, \ldots$ there are measurable $\alpha_{n}, \beta_{n}$ such that $\left(\alpha_{n}(t), \beta_{n}(t)\right)$ $\in P(t)$ a.e. in $[0,1]$ and

$$
-\alpha_{n}(t)+\eta_{n} \beta_{n}(t)=\phi_{n}(t) .
$$

Under these assumptions the class $\Omega$ composed of all absolutely continuous $z:[0,1] \rightarrow R^{2}, z=(x, y)$, such that $x(0)=0, y(0)=0, y(1)=1$ and $\dot{z}(t) \in P(t)$ a.e. in $[0,1]$ does not contain an optimal solution.

Clearly, $\Omega$ satisfies (I), (II), (III) and (IVb). From (2.40) it follows that $\dot{x}(t) \geqq 0$ a.e. in $J$ for each $(x, y) \in \Omega$. Hence $x(1) \geqq 0$ and the inf of $I(\Omega)$ is nonnegative. We shall show that it is equal to zero. For that purpose let $E_{n} \subset J$ be a measurable set such that $\int_{E_{n}} \beta_{n}(\tau) d \tau=1$. Such $E_{n}$ exist. Indeed, from (2.40), convexity of $P(t)$ 
and the positivity of $\phi_{n}$, it follows that both $\alpha_{n}(t)$ and $\beta_{n}(t)$ are positive and if $\eta_{n}<1$ then also $\alpha_{n}(t)<\beta_{n}(t)$. Thus (2.24) and the nonintegrability of $\phi_{n}$ implies that $\int_{0}^{1} \beta_{n}(t) d t=+\infty$, which in turn yields the existence of $E_{n}$. Moreover

$$
\int_{E_{n}} \alpha_{n}(t) d t<+\infty
$$

Let us put now $\dot{z}_{n}(t)=\left(\alpha_{n}(t), \beta_{n}(t)\right)$ if $t \in E_{n}$ and $(0,0)$ otherwise and $z_{n}(0)=(0,0)$. Clearly $z_{n} \in \Omega$. But from (2.43)

$$
\eta_{n} y_{n}(1)=\eta_{n} \int_{E_{n}} \beta_{n}(t) d t=\int_{E_{n}} \alpha_{n}(t) d t+\int_{E_{n}} \phi_{n}(t) d t
$$

Now $\eta_{n} \rightarrow 0, \alpha_{n}(t)$ and $\phi_{n}(t)$ are positive, and therefore $\int_{E_{n}} \alpha_{n}(t) d t \rightarrow 0$ as $n \rightarrow \infty$. But $\int_{E_{n}} \alpha_{n}(t) d t=x_{n}(1)=I\left(z_{n}\right)$. Hence $\inf _{\omega \leq \Omega} I(\omega)=0$. Suppose now that there is an $\omega^{*} \in \Omega$ such that $I\left(\omega^{*}\right)=0$. This would imply that $\dot{x}^{*}(t)=0$ a.e. in $[0,1]\left(\omega^{*}=\right.$ $\left.\left(x^{*}, y^{*}\right)\right)$. But, since $\left(\dot{x}^{*}(t), \dot{y}^{*}(t)\right) \in \Omega$ a.e. in $[0,1]$, it follows from (2.40) that $\left(\dot{x}^{*}(t), \dot{y}(t)\right)=(0,0)$ a.e. in $[0,1]$. Hence $y^{*}(1)=0=y^{*}(0)$ which contradicts the fact that $\left(x^{*}, y^{*}\right) \in \Omega$, and this completes the proof.

Notice that the sequence $\left\{z_{n}\right\}$ is convergent pointwise to a discontinuous function and $\left\{y_{n}\right\}$ does not converge uniformly.

3. Existence theorems: control system case. In this section we proceed with the discussion of the optimal control problem stated in the introduction.

Thus we consider a class $\Omega$ of solutions $(J, y, u)$ of system $(0.1)$; that is, $J$ is an interval, $y: J \rightarrow Y$ is absolutely continuous, $u: J \rightarrow E$ is measurable, and

$$
\dot{y}(t)=f(t, y(t), u(t)), \quad u(t) \in U(t, y(t)) \text { a.e. in } J .
$$

The cost function

$$
I(J, y, u)=\int_{J} g(t, y(t), u(t)) d t
$$

is a map $I: \Omega \rightarrow X$.

As before, $E, X$ and $Y$ are Euclidean spaces. We assume that an order is given in $X$ such that the positive cone $C$ is convex, closed, proper and $C-C=X$. Our goal is to give conditions implying the existence of minimal points of $I(\Omega)$.

The following assumption will be imposed upon $f, g$ and $U$ throughout this section.

Assumption 1. The maps $f: J_{0} \times Y \times E \rightarrow Y$ and $g: J_{0} \times Y \times E \rightarrow X$ are both continuous in $(y, u)$ for each fixed $t \in J_{0}=\left[a_{0}, b_{0}\right]$ and measurable in $t$ for each fixed $(y, u) \in Y \times E$. The map $U: J_{0} \times Y \rightarrow 2^{E}$ is u.s.c. in both variables $(t, y)$.

Concerning $\Omega$ we assume the following conditions:

(i) If $\left\{\left(J_{k}, y_{k}, u_{k}\right)\right\} \subset \Omega,\left(J_{*}, y_{*}, u_{*}\right)$ is a solution of (3.1) and if $\left(J_{k}, y_{k}\right) \rightarrow$ $\left(J_{*}, y_{*}\right)$ uniformly, then $\left(J_{*}, y_{*}, u_{*}\right) \in \Omega$.

(iia) There is an $M>0$ such that $\|y(t)\| \leqq M$ for each $(J, y, u) \in \Omega$ and $t$ in $J$. 
(iib) There is an $M>0$ such that for each $(J, y, u) \in \Omega$ there is $t$ in $J$ with $\|y(t)\| \leqq M$.

Put $Z=X \times Y$, and define the scalar product (thus also the norm) by

$$
\left\langle z_{1}, z_{2}\right\rangle=\left\langle x_{1}, x_{2}\right\rangle+\left\langle y_{1}, y_{2}\right\rangle,
$$

where $z_{1}=\left(x_{1}, y_{1}\right), z_{2}=\left(x_{2}, y_{2}\right)$ are two points in $Z$, and on the right-hand side of (3.3) are the scalar products in $X$ and $Y$, respectively. In this way, we may identify $X$ and $Y$ with subspaces $X \times\{0\}$ and $\{0\} \times Y$ of $Z$, respectively. As before, $X$ and $Y$ are mutually orthogonal and $Z=X \oplus Y$. We may consider also the cone $C$ as a cone in $Z$ and thus extend the order to $Z$. However, note that $\left(x_{1}, y_{1}\right) \leqq\left(x_{2}, y_{2}\right)$ if and only if $x_{1} \leqq x_{2}$ and $y_{1}=y_{2}$. The polar $C^{0}$ of $C$ in $Z$ is then

$$
C^{0}=\left\{d=\left(d_{x}, d_{y}\right) \mid\left\langle d_{x}, a\right\rangle \leqq 0 \text { for each } a \in C, d_{y} \in Y\right\} .
$$

Denote by $h$ the map from $J_{0} \times Y \times E$ into $Z$ which sends $(t, y, u)$ into $(g(t, y, u)$, $f(t, y, u)$ ). The map $h$ satisfies Assumption 1 .

The following two existence theorems correspond to Theorem 1 and Theorem 2 of the previous section.

Theorem 3. Suppose Assumption 1 holds. Assume that (i) the set

$$
Q(t, y)=\{q \in Z \mid q \geqq h(t, y, u), u \in U(t, y)\}
$$

is convex for each $(t, y) \in J_{0} \times Y$; (ii) the map $Q$ sending $(t, y)$ into $Q(t, y)$ is u.s.c. in $y$ for each fixed $t$; and (iii) for each $d \in$ int $C^{0}$ and positive $r$ there is on $J_{0}$ an integrable scalar valued function $\phi_{d}(\cdot, r)$ such that

$$
\langle d, h(t, y, u)\rangle \leqq \phi_{d}(t, r) \quad \text { if }\|y\| \leqq r \text { and } u \in U(t, y) .
$$

Under these assumptions, each nonempty class $\Omega$ of solutions of (3.1) satisfying (i) and (iia) contains an optimal solution.

THEOREM 4. In addition to all the assumptions of Theorem 4 assume that either condition (A) or (B) of Theorem 2 holds for the function $\phi_{d}(\cdot, r)$ in (3.6). Then each class $\Omega$ satisfying (i) and (iib) contains an optimal solution.

For the proof of the above theorems we will need the following extension of "Filoppov's implicit function lemma" in [3].

Proposition 4. Let $i: J \times E \rightarrow Z$ be continuous in $u \in E$ for each $t \in J$ and measurable in $t \in J$ for each $u \in E$. Let $W: J \rightarrow 2^{E}$ be u.s.c. Define

$$
Q(t)=\{z \mid z \geqq i(t, u), u \in W(t)\}
$$

and suppose there is a measurable $z: J \rightarrow Z$ such that

$$
z(t) \in Q(t) \text { a.e. in } J .
$$

Then there is a measurable $u: J \rightarrow E$ such that

$$
z(t) \geqq i(t, u(t)) \text { and } u(t) \in W(t) \text { a.e. in } J
$$


We shall only sketch the proof of Proposition 3. It is in fact very much like the case when in both (3.7) and (3.9) the inequality is replaced by equality. In the latter case the result is known (cf. [5] and [6]).

We wish to prove the existence of the function $u: J \rightarrow E$ with the property

$$
u(t) \in V(t)=\{u \in W(t) \mid i(t, u) \leqq z(t)\} .
$$

In other words $u$ is to be a measurable selection for the set valued function $V: J \rightarrow 2^{E}$. Such a selection does exist if $V$ is itself measurable; that is, if for each closed $F \subset E$, the set

$$
V^{-} F=\{t \mid v(t) \cap F \neq \varnothing\} \quad \text { is measurable }
$$

(cf. [14]). Now (3.11) is true if one shows it is true for each compact $F$, since each closed $F=\bigcup F_{k}$ where $F_{k}$ are compact and $V^{-}\left(\bigcup F_{k}\right)=\bigcup\left(V^{-} F_{k}\right)$. The set (3.11) is closed when $F$ is compact if $i$ and $z$ in (3.10) are continuous. This is so because the positive cone is closed and therefore the inequality is preserved in the limit. If $i$ and $z$ in (3.10) are not continuous but satisfy the assumption of Proposition 4, then for each $\varepsilon>0$ there is a closed subset $K \subset J$ such that the measure $\mu(J \backslash K)<\varepsilon$, and $z$ restricted to $K$ and $i$ restricted to $K \times E$ are both continuous. The first is the celebrated Lusin's theorem, the second is an extension of a result due to Scorza Dragoni (cf. Jacobs [6, Corollary 2.3]). But this means that the set (3.11) can be approximated as closely as desired by a closed set. Hence it is measurable.

Proof of Theorems 3 and 4. Consider the orientor field

$$
\dot{z}(t) \in Q(t, y(t))
$$

where $Q$ is given by (3.5). Let $\bar{\Omega}$ be the set of solutions of (3.12) such that $(J, x, y)$ $\in \bar{\Omega}$ if and only if $x(a)=0$.

Now there is a measurable $u: J \rightarrow E$ such that $(J, y, u)$ satisfies (3.1) and belongs to $\Omega$. If $(J, y, u) \in \Omega$, and $x(t)=\int_{a}^{t} g(\tau, y(\tau), u(\tau)) d \tau$, then it is clear that $(J, x, y)$ $\in \bar{\Omega}$ since $\dot{x}(t)+\dot{y}(t)=h(t, y(t), u(t)) \in Q(t, y(t))$. Thus we have an $e: \Omega \rightarrow \bar{\Omega}$ which maps $(J, y, u) \rightarrow(J, x, y)$ where $x(t)$ is defined as above. This map has the property

$$
I(\omega)=\bar{I}(e(\omega)) \text { for each } \omega \in \Omega,
$$

where $\bar{I}: \bar{\Omega} \rightarrow X$ and $\bar{I}(J, x, y)=x(b)$.

On the other hand, by Proposition 4, for each $\bar{\omega}=(J, x, y) \in \bar{\Omega}$ there is a measurable $u: J \rightarrow E$ such that (3.9) holds, where $i(t, u)=h(t, y(t), u)=(g(t, y(t), u)$, $f(t, y(t), u)$ ) and $W(t)=U(t, y(t))$. But the first inequality of (3.9) means that $\dot{y}(t)=f(t, y(t), u(t))$ and $\dot{x}(t) \geqq g(t, y(t), u(t))$. Thus $(J, y, u)$ is a solution of (3.1) belonging to $\Omega$ and $x(b) \geqq \int_{J} g(t, y(t), u(t)) d t$. Hence $\bar{\Omega}$ can be mapped into $\Omega$, say by a map $\bar{e}$, with the property that

$$
I(\bar{e}(\omega)) \leqq \bar{I}(\omega) \text { for each } \omega \in \bar{\Omega} .
$$

It follows from (3.13) and (3.14) that each minimal point of $\bar{I}(\bar{\Omega})$ is a minimal point of $I(\Omega)$. Indeed, let $p \in \vec{I}(\bar{\Omega})$ be minimal. By (3.14) there is $q \in I(\Omega)$ such that 
$q \leqq p$. We claim that $q=p$ and $q$ is minimal for $I(\Omega)$. Let $q_{1} \in I(\Omega)$ and $q_{1} \leqq q$. Then by (3.13) $q_{1} \in \bar{I}(\bar{\Omega})$ and $q_{1} \leqq q \leqq p$. But $p$ is minimal, and hence $q_{1}=p$. Therefore $q_{1}=q=p$ and $q$ is minimal for $I(\Omega)$.

In order to prove Theorem 3 (or 4 ) it suffices to show that $\bar{\Omega}$ and $Q$ defined by (3.5) satisfy the conditions of Theorem 1 (or 2). By definition, $\bar{\Omega}$ satisfies (I) and (II). Condition (III) follows easily from (i) and Proposition 4. Finally (IVa) and (IVb) follow from (iia) and (iib), respectively.

The assumption (2.4) concerning $Q$ is readily satisfied since $Q$ depends only on $y$ and does not depend on $x . Q$ is u.s.c. by assumption. It is clear by (3.5) that the asymptotic cone $C_{Q(t, y)}$ contains $C$. Suppose that for some $(t, y)$ the cone $C$ is a proper subset of $C_{Q(t, y)}$ and let $a \in C_{Q(t, y)} \mid C$. Then there is $d \in$ int $C^{0}$ such that $\langle d, a\rangle>0$. But for each $q \in Q(t, y)$ and $\lambda>0, q+\lambda a \in Q(t, y)$. This and (3.5) contradict (3.6). Hence (2.3) holds. Finally (2.5) follows from (3.6) and (3.5), and conditions (A) and (B) are the same in both Theorem 2 and Theorem 4. Thus the proof is completed.

REMARK 1. It follows from Assumption 1 that any change of either $f$ or $g$ or both (and thus also $Q$ defined by (3.5)) on a set $N \times Y \times E$ where $N \subset J_{0}$ has measure zero is irrelevant and does not affect the conclusion of both Theorems 3 and 4 . On the other hand, from Proposition 1' it follows that if $Q$ is defined by (3.5) and $\phi_{d}(t, r)$ with $(t, r)$ fixed is finite for $d$ from a dense subset of int $C^{0}$, then it has to be finite for each $d \in$ int $C^{0}$. Therefore there is no loss of generality from assuming that (3.6) holds everywhere in $J$ or that $\phi_{d}(t, r)$ is finite for each $t$ (compare this with the corollary of $\S 2$ ).

REMARK 2. The purpose of this remark is to contrast our Theorems 3 and 4 with Cesari's analogous result [1, Existence Theorem I, p. 390]. For that purpose the reader can think about $X$ being one dimensional, $C$ as positive half $x$-axis in $Z$, int $C^{0}=\{(x, y) \in Z \mid x<0\}$. This is indeed the case considered by Cesari. In the case corresponding to Theorem 3 ( $\Omega$ satisfies (iia)), Cesari assumes the so-called "growth condition" on each bounded subset of $Y$; that is, for each bounded subset $B$ of $Y$ there exist a continuous function $\Phi: R_{+} \rightarrow R$ such that $\Phi(\zeta) / \zeta \rightarrow \infty$ as $\zeta \rightarrow \infty$ and two positive constants $G, H$ such that $g(t, y, u) \geqq \Phi(\|u\|)$ and $\|f(t, y, u)\| \leqq G+H\|u\|$ for $(t, y) \in J \times B$ and $u \in U(t, y)$. It is easy to check that this implies (3.6) with $\phi_{d}$ independent of $t$. Indeed, if $d \in$ int $C^{0}, d=\left(d_{x}, d_{y}\right)$, then $d_{x} q(t, y, u)+\left\langle d_{y}, f(t, y, u)\right\rangle \leqq+d_{x} \Phi(\|u\|)+\left\|d_{y}\right\|(G+H\|u\|)$ (remember that $\left.d_{x}<0\right)$. Since the latter sum tends to $-\infty$ as $\|u\| \rightarrow+\infty$ it is bounded from above. The assumptions concerning the set $Q$ are the same with the exception that Cesari assumed condition (2.8) with respect to both variables while we assume $Q$ is u.s.c. in a weaker sense and only with respect to one variable. Similarly, $f$ and $g$ are also assumed in [1] to be continuous in $t$.

Concerning the unbounded case (condition (iib)), besides the growth condition it is assumed in [1] that there are constants $G_{1}$ and $H_{1}$ such that $g(t, y, u) \geqq$ $G_{1}\|f(t, y, u)\|$ if $\|y\|>H_{1}, u \in U(t, y)$. Those two conditions imply that the function 
$\phi_{d}$ in (3.6) can be taken as constant if $d=\left(\eta d_{x}, d_{d}\right), d_{x}<0$, and $\eta$ is small enough. Therefore this is taken care of by our Theorem 4 case (A). There is no counterpart in [1] to our Theorem 4 case (B). In other words, Cesari always assumes 'almost explicitly' an a priori bound for the infimum of the cost functional.

As follows from the proof of Theorem 2 (see also the discussion of the example in $\S 2$ ) conditions (A) and (B) are to guarantee that the subclass

$$
\Omega_{0}=\left\{\omega \in \Omega \mid I(\omega) \leqq I\left(\omega_{0}\right)\right\}
$$

of $\Omega$ satisfying (IVb) (or (iib)) satisfies (IVa). This can be assured by different kinds of conditions not connected with the bound in (3.6). These are the types of conditions which supply an a priori bound for a solution of (3.1) satisfying a fixed initial condition (independent of $g$ ). We omit details here.

ExAmple. The problem we considered contains as a special case the classical Lagrange problem in the calculus of variations which corresponds to the case when $f(t, y, u) \equiv u$ and $U(t, y)=E=Y$. The convexity of the set $Q(t, y)$ is now equivalent to convexity of $g$ in $u$. An extension of the existence theorems of Nagumo [13], McShane [12], and Tonelli [16] can be formulated. However we restrict ourselves to an example related to the examples at the end of $\S 2$.

Suppose we wish to minimize the functional

$$
\int_{0}^{t}\left[\alpha(t, y(t)) \dot{y}^{2}(t)+\gamma(t, y(t))\right] d t
$$

in the class $\Omega$ of absolutely continuous functions satisfying the boundary value condition:

$$
y(0)=1, \quad y(t)=0, \quad t \leqq 1 .
$$

If $\alpha(t, y)=t$ and $\gamma \equiv 0$, an optimal solution does not exist. In fact in this case the bound in (3.6) (compare with (2.32)) is a constant times $t^{-1}$ and therefore is not integrable (cf. Example 2 of $\S 2$ ). However, if $\alpha \geqq t^{1-\varepsilon}$, then an optimal solution exists even with $\gamma(t, y)=\gamma_{0}(t)+\gamma_{1}(t) y$ provided that both $\gamma_{0}$ and $\gamma_{1}$ are integrable on $[0,1]$.

\section{REFERENCES}

1. L. Cesari, Existence theorems for weak and usual optimal solutions in Lagrange problems with unilateral constraints. I, Trans. Amer. Math. Soc. 124 (1966), 369-412.

2. N. O. DaCunha and E. Polak, "Constrained minimization under vector-valued criteria in linear topological spaces" in Mathematical theory of control, Academic Press, New York, 1967, pp. 96-108.

3. A. F. Filippov, On certain questions in the theory of optimal control, Vestnik Moskov. Univ. Ser. I Mat. Meh. 2 (1959), 25-32.

4. - Differential equations with many-valued discontinuous right-hand side, Dokl. Akad. Nauk SSSR 151 (1963), 65--68=Soviet Math. Dokl. 4 (1963), 941-945.

5. M. Q. Jacobs, Attainable sets in systems with unbounded controls, J. Differential Equations (to appear).

6. - Remarks on some recent extensions of Filippov's implicit functions lemma, SIAM J. Control 5 (1967), 622-627. 
7. V. Klee and C. Olech, Characterization of a class of convex sets, Math. Scand. 20 (1967).

8. K. Kuratowski, Les functions semi-continues dans l'espace des ensembles fermés, Fund. Math. 18 (1932), 148-166.

9. K. Kuratowski and C. Ryll-Nardzewski, A general theorem on selectors, Bull. Acad. Polon. Sci. Sér. Math. Astronom. Phys. 13 (1965), 397-403.

10. A. Lasota and C. Olech, On the closedness of the set of trajectories of a control system, Bull. Acad. Polon. Sci. Sér. Sci. Math. Astronom. Phys. 14 (1966), 615-621.

11. E. B. Lee and L. Markus, Optimal control for nonlinear processes, Arch. Rational Mech. Anal. 8 (1961), 36-58.

12. E. J. McShane, Existence theorems for ordinary problems of the calculus of variations, Ann. Scuola Norm Sup. Pisa 3 (1934), 181-211.

13. M. Nagumo, Über die gleichmassige Summierbarkeit und ihre Anwendung auf ein Variations-problem, Japan. J. Math. 6 (1929), 178-182.

14. A. Pliś, Measurable orientor fields, Bull. Acad. Polon. Sci. Sér, Sci. Math. Astronom. Phys. 13 (1965), 565-569.

15. E. O. Roxin, The existence of optimal controls, Michigan Math. J. 9 (1962), 109-119.

16. L. Tonneli, Sugli integrali del calcolo delle variazioni in forma ordinaria, Ann. Scuola Norm Sup. Pisa 3 (1934), 401-450; Opere scelte. vol. 3, Edizioni Cremonese, Rome, 1962, pp. 192-254.

17. T. Ważewski, "On an optimal control problem" in Differential equations and their applications (Proc. Conf., Prague, 1962), Academic Press, New York, 1963, pp. 229-242.

BroWN UNIVERSITY,

Providence, Rhode IsLAND

Polish ACAdemy of Sciences,

Kraków, Poland 